\title{
Spatial Attention and Memory Versus Motor Preparation: Premotor Cortex Involvement as Revealed by fMRI
}

\author{
STÉPHANE R. SIMON, ${ }^{1}$ MARTINE MEUNIER, ${ }^{2}$ LOŸS PIETTRE, ${ }^{1}$ ANNA M. BERARDI, ${ }^{1}$ \\ CHRISTOPH M. SEGEBARTH, ${ }^{1}$ AND DRISS BOUSSAOUD ${ }^{2}$ \\ ${ }^{1}$ Unité Mixte Université Joseph Fourier, Institut National de la Santé et de la Recherche Médicale 438, Laboratoire de \\ Recherche Correspondant Commissariat à L'Energie Atomique, 38043 Grenoble, France; and ${ }^{2}$ Institut des Sciences \\ Cognitives, Centre National de la Recherche Scientifique et Université Claude Bernard, Unité Mixte de Recherche 5015, \\ 69675 Bron, France
}

Received 26 November 2001; accepted in final form 24 June 2002

\begin{abstract}
Simon, Stéphane R., Martine Meunier, Lö̈s Piettre, Anna M. Berardi, Christoph M. Segebarth, and Driss Boussaoud. Spatial attention and memory versus motor preparation: premotor cortex involvement as revealed by fMRI. J Neurophysiol 88: 2047-2057, 2002; 10.1152/jn.00965.2001. Recent studies in both monkeys and humans indicate that the dorsal premotor cortex participates in spatial attention and working memory, in addition to its well known role in movement planning and execution. One important question is whether these functions overlap or are segregated within this frontal area. Singlecell recordings in monkeys suggest a relative specialization of the rostral portion of dorsal premotor cortex for attention and/or memory and of the caudal region for motor preparation. To test whether this possibility also holds true in humans, we used functional magnetic resonance imaging (fMRI) to compare, in the same set of subjects, brain activation related to strong spatial attention and memory demands to that elicited by long motor preparatory periods. The behavioral protocol was based on a task that had proved effective for dissociating neuronal properties related to these two functions in the monkey brain. The principle of the monkey task was that a first cue guided the focus of spatial attention and memory, whereas a second one instructed an arm movement. Based on this principle, two tasks were developed. One maximized spatial attention and memory demands by presenting long series of stimuli $(4,8$, or 12$)$ before the motor instructional cue, whereas the other extended the motor preparation phase by imposing long and variable delays (1-5.5 s) between the onset of the instructional cue and movement execution. The two tasks and their respective control conditions were arranged in two blocked-design sequences. The results indicate that the brain networks underlying the two functional domains overlap in the caudate nucleus and presupplementary motor area, and possibly in lateral prefrontal cortex as well, but involve different dorsal premotor fields. Motor preparation primarily recruited a dorsal premotor area located caudally, within the precentral gyrus (together with the supplementary motor area), whereas spatial attention and memory preferentially activated a more rostral site, in and anterior to the precentral sulcus (in addition to the posterior parietal cortex). These findings strengthen the idea that the primate dorsal premotor cortex contributes to both motor and nonmotor processes. Moreover, they corroborate emerging evidence from monkey physiology suggesting a relative functional segregation within this cortex, with attention to short-term storage of visuospatial information engaging a more rostral region than motor preparation.
\end{abstract}

Address for reprint requests: D. Boussaoud, Institut des Sciences Cognitives, 67 boulevard Pinel, 69675 Bron cedex, France (E-mail: boussaoud@isc.cnrs.fr).

\section{N T R O D U C T I O N}

There is a general agreement that the dorsal premotor cortex (PMd) in primates plays a major role in the selection, planning, and execution of voluntary movements (for reviews, see Caminiti et al. 1998; Wise et al. 1997). This idea was first derived from observations of brain-damaged patients and from monkeys with experimental lesions (Halsband and Freund 1990; Kurata and Hoffman 1994; Passingham 1993; Petrides 1986). It has been supported by numerous physiological studies in monkeys (Caminiti et al. 1991; Crammond and Kalaska 2000; di Pellegrino and Wise 1993; Fu et al. 1995; Kurata and Hoffman 1994; Riehle and Requin 1989; Weinrich and Wise 1982) and recently confirmed by brain imaging investigations in humans (Deiber et al. 1996, 1997; Grafton et al. 1998; Kawashima et al. 1994; Lee et al. 1999; Richter et al. 1997; Toni et al. 1999, 2001; van Oostende et al. 1997).

Accumulating evidence indicates, however, that in addition to its well-established motor function, the primate premotor cortex plays a role in nonmotor processes such as spatial attention and working memory. On the one hand, human imaging studies targeting either spatial attention or working memory have repeatedly reported activation in the premotor cortex (e.g., Corbetta et al. 1998; Coull and Nobre 1998; Courtney et al. 1998; D’Esposito et al. 1998; Kawashima et al. 1998; Petit et al. 1996, 1998; Stern et al. 2000). On the other hand, neurophysiological experiments in monkeys have identified in PMd a substantial proportion of cells whose activity reflects spatial attention and/or memory in addition to the large population of cells with motor preparatory properties (Boussaoud and Kermadi 1997; Boussaoud and Wise 1993a,b; di Pellegrino and Wise 1993; Lebedev and Wise 2001).

In light of these data, one important question is whether attentional/mnemonic and motor functions overlap within PMd, or whether they are carried out by distinct subregions. Recent neurophysiological findings in monkeys (Boussaoud 2001; Lebedev and Wise 2001) suggest that attention/memory related activity is more frequent in the rostral portion of PMd (PMdr), whereas motor preparatory activity is predominant in

The costs of publication of this article were defrayed in part by the payment of page charges. The article must therefore be hereby marked "advertisement" in accordance with 18 U.S.C. Section 1734 solely to indicate this fact. 
the caudal portion (PMdc). By contrast, no experiment in humans has directly compared premotor contribution to these functions in the same group of subjects. To this aim, we used functional magnetic resonance imaging (fMRI) in healthy subjects and a behavioral protocol adapted from a task designed in an earlier series of studies (Boussaoud 2001; Boussaoud and Kermadi 1997; Boussaoud and Wise 1993a,b) to dissociate attentional/mnemonic from motor preparatory neuronal activity in the monkey brain.

The principle of the monkey task was that the animal had first to attend to and remember the location of a spatial attentional/mnemonic (SAM) stimulus, and a motor instructional/ conditional (MIC) cue appeared later to guide the direction of its subsequent arm movement. In some trials, the SAM stimulus and the MIC cue were physically identical-identical in all spatial coordinate systems and visual attributes-but differed in their behavioral significance. The first affected spatial attention and/or memory, whereas the second instructed a motor act. Single-cell recordings during these trials (Boussaoud and Kermadi 1997; Boussaoud and Wise 1993a,b) demonstrated that although the activity of numerous premotor neurons $(70 \%)$ expectedly reflected motor preparation, a substantial proportion $(30 \%)$ of premotor cells were specifically active in relation with spatial attention/memory. Furthermore, the distribution of SAM-related and MIC-related neurons within PMd was found to differ between rostral and caudal divisions of PMd (Boussaoud 2001), with the former more frequent in PMdr (38\% vs. $23 \%$ ) and the latter more predominant in PMdc (65\% vs. 38\%).

Based on the SAM/MIC principle of the monkey task, two paradigms were developed for this study. One was designed to maximize spatial attention and memory demands by increasing the number of SAM stimuli preceding the MIC cue, and the other to extend the motor preparation phase by imposing long and variable delays between MIC presentation and movement execution. The two tasks and their respective baselines were arranged in two blocked-design sequences termed the spatial attention and memory paradigm (SAMP) and motor preparation paradigm (MPP). To directly compare the premotor regions engaged in these two functional domains, a single group of well-trained subjects consecutively performed the two paradigms while undergoing functional scanning. On the basis of neurophysiological data in monkeys, we hypothesized that spatial attention/memory and motor preparation will engage distinct regions within PMd.

Like the monkey task, the present paradigms did not involve eye movements; SAM and MIC cues appeared at the periphery of the visual field while subjects maintained their gaze on a central fixation point. However, the human frontal eye field (FEF) is known to be activated even by attentional tasks devoid of eye movements (Beauchamp et al. 2001; Corbetta et al. 1998; Nobre et al. 2000), and unlike its monkey counterpart, is thought to lie within rather than rostral to the premotor strip (Paus 1996; Tehovnik et al. 2000). We therefore submitted a subset of subjects to an oculomotor control paradigm to evaluate FEF contribution to the activation pattern induced by our spatial attention and memory paradigm. The data reported in this paper have been presented earlier in abstract form (Simon et al. 2000, 2001).

\section{METHODS}

\section{Subjects and setup}

Ten healthy volunteers were studied ( 6 males, 4 females; mean age $29 \pm 10 \mathrm{yr}$ ). All subjects were strongly right-handed, as assessed by a French adaptation of the Edinburgh Handedness Scale (Oldfield 1971). Experiments were conducted with the understanding and consent of each subject.

Visual stimuli were generated by a Power Macintosh 9600 computer (Apple, Cupertino, CA) using Psyscope V1.2.2 software (Carnegie Mellon Department of Psychology; Cohen et al. 1993). They were presented using a video projector (Eiki LC 6000; Eiki Industrial Co. Ltd., Osaka, Japan), a projection screen fixed on the back of the magnet, and a mirror placed atop of the head coil. The SAM stimulus consisted of one white square $\left(1.4^{\circ} \times 1.4^{\circ}\right)$ presented at one of eight predefined locations equidistant from the screen center ( 2 per quarter of the visual field, positioned at $29^{\circ}$ and $44^{\circ}$ with respect to the vertical meridian). The MIC cue was composed of two adjacent squares (with a collinear diagonal, see Fig. 1), one green and one red $\left(1.4^{\circ} \times 1.4^{\circ} \mathrm{each}\right)$, one of which was at the location of the immediately preceding SAM stimulus. A central white cross $\left(0.7^{\circ} \times 0.7^{\circ}\right)$ served as a fixation point, except during MIC presentation. All stimuli were presented on a dark background. Subjects responded by pressing one of two keys positioned under their right index and middle fingers. The computer was used for both stimulus presentation and data acquisition (key presses and response times) in real-time.

\section{SAMP and MPP}

Each of the two "block" paradigms alternated three task and three control epochs of equal duration and lasted a total of $7.8 \mathrm{~min}$. Switching between epochs was signaled by a 1-s text instruction. All variable parameters (colors, locations, delays, and responses) followed a counterbalanced pseudo-random order across trials ensuring matching sensory-motor demands in the task and control conditions of each paradigm. In all testing conditions, subjects were required to maintain their gaze on the central fixation cross during the entire duration of an epoch, and correct responses were guided by the same arbitrary associative rule, where red instructed to respond with the index finger and green with the middle finger.

SAMP. For both SAMP task and control conditions (Fig. 1A), a series of 4,8 , or 12 SAM stimuli was presented for $0.5 \mathrm{~s}$, separated by variable delays of $1.25,1.5,1.75$, or $2 \mathrm{~s}$; then, the MIC cue was presented for $1.5 \mathrm{~s}$, and the white fixation cross simultaneously turned either green or red. The actual lengths of the series of SAM stimuli were unknown to the subject. For SAMP task condition, subjects had to determine which of the two squares composing MIC occupied the location of the last SAM stimulus, identify its color, and respond according to the arbitrary rule indicated above. For SAMP control condition, subjects were asked to focus on the white fixation cross and respond as soon as it turned green or red, following the same conditional rule. Therefore the sensory input, motor output, gaze direction, and associative rule guiding behavior were identical in SAMP task and control. The difference was that, during the task, the subjects had to attend to and remember each of the successive peripheral positions of the SAM stimulus (while fixating the central cross) and form a spatial matching judgment, whereas during the control, attention was focused on the center of the screen, and spatial memory was not required. Each epoch comprised four trials, one with 4, two with 8, and one with 12 SAM stimuli, arranged in a counterbalanced order across epochs. As a result, the amount of scanning time devoted to spatial attention/memory in the task condition was approximately 12-fold longer than that devoted to motor preparation.

MOTOR PREPARATION PARADIGM (MPP). For both MPP task and control conditions (Fig. $1 B$ ), a single SAM stimulus was presented for $0.25 \mathrm{~s}$, followed by a 0.25 -s delay; then, the MIC cue was 


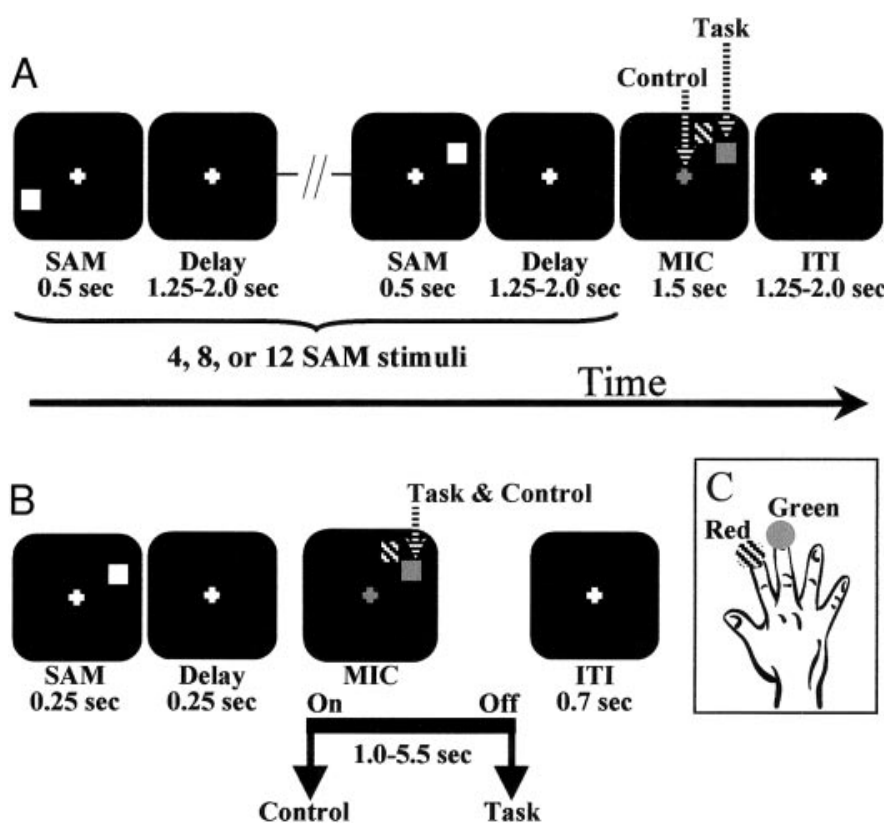

FIG. 1. Behavioral paradigms. $A$ and $B$ : example of a trial for the task and control conditions of each paradigm. In all conditions, subjects were asked to maintain their gaze on a central fixation cross and use a single conditional rule associating red with an index finger response and green with a middle finger response $(C)$. Each panel shows the status of the video screen at a point in the trial. Time progresses from left to right, as represented by the arrow (center). In each screen, the cross represents the fixation point and the small squares indicate the location of a stimulus. A: spatial attention and memory paradigm (SAMP). A white square served as the spatial attentional/mnemonic stimulus (SAM). Each presentation of SAM stimulus was followed by a variable delay. At the end of the series, 2 squares, 1 red and 1 green [motor instructional/ conditional cue (MIC)] appeared, and the fixation cross turned either green or red. For the task, the correct response was determined by the color of the MIC square that occupied the position of the last SAM stimulus (green in this example). For the control, the response was determined by the color of the fixation cross accompanying MIC presentation (green in this case). B: motor preparation paradigm (MPP). A single SAM stimulus was followed by a fixed delay. Then the MIC cue was presented for variable durations together with a green or red fixation cross. For both task and control, responses were determined by the color of the square that occupied the location of the SAM stimulus (green in this example). The difference was that, for the task, the subjects were asked to withhold their response until MIC offset (and concomitant return of the white cross), whereas, for the control, they were asked to respond immediately after MIC onset.

presented for variable periods of time $(1,2,3,3.5,4,4.5,5$, or $5.5 \mathrm{~s}$ ), followed by a 0.7 -s inter-trial interval. The fixation cross was either green or red during MIC presentation, turning white again at MIC offset. For both MPP task and control, responses were determined by the color of the MIC square that occupied the position of the SAM stimulus. For the task, subjects were told to select and prepare their movement at MIC onset, but to withhold its execution until MIC offset (and concomitant return of the white cross, this additional go signal allowing covert attention to shift back to the screen center during preparatory periods); in addition, subjects were explicitly instructed not to actually move their finger before the go signal and to refrain from verbally rehearsing the associative rule or the name of the correct color or finger. For the control, subjects were asked to respond immediately after MIC onset and to then resume attending to the fixation cross. Thus the perceptual input, motor output, associative rule, gaze direction, and attentional shifts were identical in the two MPP conditions. The difference was that the motor preparation phase was much longer during the task than during the control. Sixteen trials were performed per epoch. Thus the amount of scanning time devoted to motor preparation in the task condition was approximately 8 -fold longer than that devoted to spatial attention/memory.

Prior to scanning, all subjects were trained on the different tasks until they reached a performance of $\geq 90 \%$ correct responses, associated with stable reaction times. Feedback was provided during the practice sessions, but not during scanning.

\section{Oculomotor control paradigm}

Three of the 10 subjects were submitted to an oculomotor paradigm in a separate "block," alternating three task and three control epochs for a total of $7.8 \mathrm{~min}$. This paradigm was strictly identical to the SAMP paradigm described above except that, during the task condition, subjects were asked to make an ocular saccade to each SAM stimulus and resume fixation of the central cross.

\section{MR acquisition}

For all subjects, two fMRI examinations were performed during a single experimental session, one for SAMP and one for MPP, with the order of the two paradigms counterbalanced across subjects. Three of the 10 subjects were submitted in addition, at the end of the scanning session, to the oculomotor paradigm. Measurements were performed at 1.5 Tesla on a clinical MR imager (Philips NT, Best, The Netherlands). The body coil was used for excitation while the head coil was used for detection. A volume composed of 25 slices (slice thickness = $4 \mathrm{~mm}$ ) parallel to the anterior commissure-posterior commissure (AC-PC) axis was measured 24 times during each epoch. The volume encompassed the whole brain except the posterior lobe of the cerebellum. Positioning of the volume was performed on scout images acquired in the sagittal plane. The volume was measured twice in a dummy fashion prior to testing, so that system stability could be achieved. The functional scans were performed by means of a gradient-recalled echo, echo-planar imaging MR sequence. T2*-weighted images were acquired. The major MR sequence parameters were as follows: repetition time $(\mathrm{RT})=3700 \mathrm{~ms}$, echo time $(\mathrm{ET})=45 \mathrm{~ms}$, pulse angle $=90^{\circ}$, acquisition matrix $=64 \cdot 64$, field-of-view $=256$. $256 \mathrm{~mm}^{2}$, and in-plane resolution $=4 \mathrm{~mm}$. Prior to acquisition, a chemically selective radio frequency (RF) pulse was applied to suppress the signals from fat. Finally, a high-resolution three-dimensional T1-weighted MR scan was acquired to provide anatomical information about the volume examined functionally.

\section{Image processing and statistical analysis}

Data analysis was performed using statistical parametric mapping (SPM)-96 software (Wellcome Department of Cognitive Neurology, London, UK; see Friston et al. 1995a) running on a Unix workstation under the MATLAB environment (Mathworks, Sherbon, MA). MR images were subjected to three preprocessing steps. All images within a functional scan were first realigned by means of a rigid body transformation for motion correction (Friston et al. 1995b). Then, the anatomical volume was spatially normalized using as template a representative brain from the MNI series (Montreal Neurological Institute, Quebec, Canada; Evans et al. 1993) and linear transformations (Friston et al. 1995b); these normalization parameters were subsequently applied to the functional images. Finally, the functional images were spatially smoothed with an isotropic Gaussian kernel of $6 \mathrm{~mm}$ width.

Statistical contrasts were performed both individually for each subject and for the overall group of subjects using the general linear model (Friston et al. 1995a; Worsley and Friston 1995; Worsley et al. 1992). Clusters of activated voxels were then identified on the basis of the intensity of the individual responses and the spatial extent of the clusters Statistical significance thresholds were established at $P=$ 0.001 for individual voxels and at $P=0.05$ (corrected for multiple comparisons) for cluster size (corresponding to a minimum of 11 
voxels). Single subtraction contrasts were first computed separately for SAMP and MPP to localize brain regions engaged in the spatial attention/memory and motor preparation tasks relative to their respective controls. To identify the brain areas that were equally active in both tasks, a conjunction analysis was performed (Price and Friston 1997). This SPM conjunction analysis summed the two paradigms [(SAMP task + MPP task) - (SAMP control + MPP control) $]$ and removed the voxels, showing a significant interaction across paradigms. The resulting activation map was masked $(P<0.001)$ with the two single (task - control) contrasts to ensure that all voxels identified by the conjunction analysis were significantly active in both paradigms. To identify the areas preferentially involved in SAMP, an interaction analysis was performed. This analysis summed the two paradigms and retained the voxels for which the (task - control) contrast was significantly larger for SAMP than for MPP. The resulting activation map was masked by SAMP contrast to ensure that effects due to MPP negative activation were excluded. A similar interaction and masking procedure was used to identify the brain regions that were preferentially involved in MPP. Two additional analyses were carried out for the three subjects who performed the oculomotor control paradigm. First, we used the interaction and masking procedure to identify the areas whose activation was significantly greater for the oculomotor control paradigm than for SAMP. Then, a single subtraction contrast was performed to determine the pattern of activation elicited by SAMP in this particular subset of subjects. For all analyses, coordinates of the activated brain areas in the MNI system of reference were transformed into the Talairach and Tournoux stereotaxic space (Talairach and Tournoux 1988), using the following equations: $x_{\mathrm{T}}=0.88 x_{\mathrm{MNI}}+0.8 ; y_{\mathrm{T}}=0.97 y_{\mathrm{MNI}}-3.32$; $z_{\mathrm{T}}=0.05 y_{\mathrm{MNI}}+0.88 z_{\mathrm{MNI}}-0.44$.

\section{R E S U L T S}

\section{Behavioral data}

The accuracy and reaction time data recorded during fMRI examination are provided for each paradigm and each condition in Table 1 . The 10 subjects included in the study scored above $90 \%$ correct responses in all four testing conditions. A $2 \times 2$ repeated measures ANOVA on accuracy scores yielded a marginal paradigm effect $[F(1,9)=5.2, P=0.05]$, reflecting the subjects' slightly reduced accuracy in SAMP task relative to MPP task [paired $t$-test with Bonferroni adjusted probability $\left.\left(p_{\mathrm{B}}\right): t(9)=2.6, p_{\mathrm{B}}=0.17\right]$. However, the lack of significant condition or interaction effect indicated that, within each paradigm, subjects were comparably accurate for the task and control conditions (hence, fMRI activation patterns are unlikely due to different difficulty levels across conditions). A $2 \times 2$ repeated measures ANOVA on reaction times revealed a significant paradigm $\times$ condition interaction $[F(1,9)=162$, $P<0.001]$, which confirmed within-paradigm intended differences. Namely, for SAMP, reaction times were longer for

TABLE 1. Mean percentage correct responses (\%) and reaction time $(R T)$ under the four testing conditions

\begin{tabular}{lcc}
\hline \hline \multicolumn{1}{c}{ Condition } & $\% \pm \mathrm{SD}$ & $\mathrm{RT} \pm \mathrm{SD}(\mathrm{ms})$ \\
\hline SAMP task & $92.5 \pm 6.1$ & $782 \pm 102^{*}$ \\
SAMP control & $95.0 \pm 7.0$ & $645 \pm 85$ \\
MPP task & $97.7 \pm 2.5$ & $307 \pm 22^{*}$ \\
MPP control & $95.6 \pm 4.6$ & $590 \pm 57$ \\
\hline
\end{tabular}

* Significant differences between task and control conditions as revealed by paired $t$-tests with Bonferroni adjusted probabilities, both $P<0.001$. SAMP, spatial attention and memory paradigm; MPP, motor preparation paradigm. the task than for the control $\left[t(9)=-8.4, p_{\mathrm{B}}<0.001\right]$, since only the former required a spatial matching judgment before movement execution. The reverse was true for MPP $[t(9)=$ $\left.13.7, p_{\mathrm{B}}<0.001\right]$, thereby indicating that the subjects did take advantage of the extended delays to prepare movement execution during the task.

\section{fMRI activation data}

Brain regions engaged by the spatial attention/memory and motor preparation tasks relative to their respective controls are illustrated in Fig. $2 A$ and listed in Tables 2 and 3, respectively. Direct comparison of the premotor regions recruited by these two functional domains is provided by the results of the conjunction and interaction analyses, which are listed in Table 4 and illustrated, respectively, in Fig. 2, $B$ and $C-D$. Finally, lateral premotor activation in the three subjects who performed the spatial attention and memory task both without (SAMP) and with (oculomotor control paradigm) eye movements, is reported in Table 5 and Fig. $2 E$.

SAMP AND MPP MAIN EFFECTS. The SAMP (task - control) contrast revealed a bilateral cortical network of activation involving the lateral premotor cortex (in the superior frontal and precentral sulci), medial premotor cortex [rostral to the vertical plane passing through the anterior commissure (VAC)], and inferior prefrontal cortex, as well as an extensive portion of the posterior parietal cortex (intraparietal sulcus and precuneus). By contrast, the cortical network outlined by the MPP subtraction was restricted to the frontal lobe, including the lateral premotor cortex bilaterally (posterior precentral gyrus), left primary motor cortex (M1), medial premotor cortex (both rostral and caudal to VAC), and the right middle and inferior prefrontal cortex. Subcortical activation was observed bilaterally in the caudate nucleus for both SAMP and MPP contrasts and in the right thalamus for MPP. An additional ventromedial cluster of activated voxels, centered on the amygdala, was found for MPP. This cluster should, however, be considered with great caution since the AC-PC low-spatial resolution echoplanar imaging used here is known to result in artifactual activation in the ventromedial region of the brain (due to the vicinity of air-filled bony cavities at the skull base; Merboldt et al. 2001).

Superimposition of SAMP and MPP activation maps showed some overlap in the caudate nucleus, medial premotor cortex, and possibly in lateral prefrontal cortex as well, but not in the dorsal premotor fields (Fig. $2 A$, lateral views). In both hemispheres, SAMP activation peak in dorsal premotor cortex was located $8 \mathrm{~mm}$ anterior to MPP peak (Table 4). This rostro-caudal segregation was confirmed by the results of the conjunction and interaction analyses.

CONJUNCTION ANALYSIS: BRAIN REGIONS SIMILARLY ENGAGED IN BOTH PARADIGMS. According to this analysis, no lateral premotor area was similarly recruited by the spatial attention/ memory and motor preparation tasks. Only two regions were identified as equally active in the two paradigms: the right caudate nucleus and a midline area involving the medial frontal gyrus and extending in the underlying cingulate sulcus, at the rostral part of the activated foci. The site in the medial frontal gyrus was located rostral to the VAC plane, thereby coinciding with presupplementary motor area (pre-SMA; Picard and Strick 1996). 

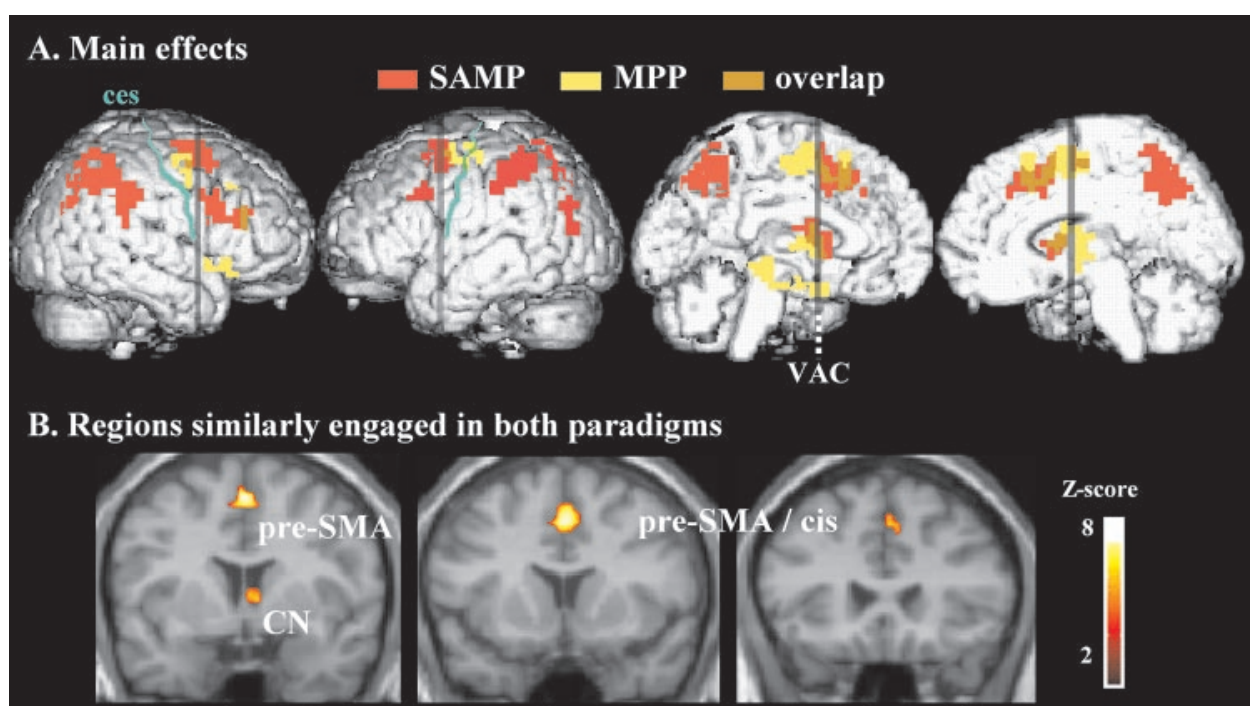

$\mathrm{y}=4 \mathrm{~mm}(1)$

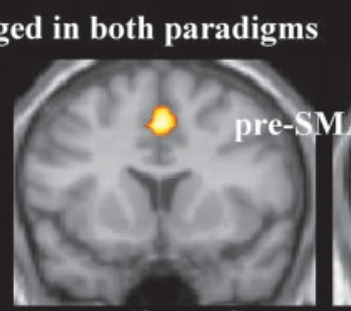

$\mathrm{y}=12 \mathrm{~mm}(8)$

\begin{abstract}
$\mathrm{y}=24 \mathrm{~mm}(20)$
\end{abstract}
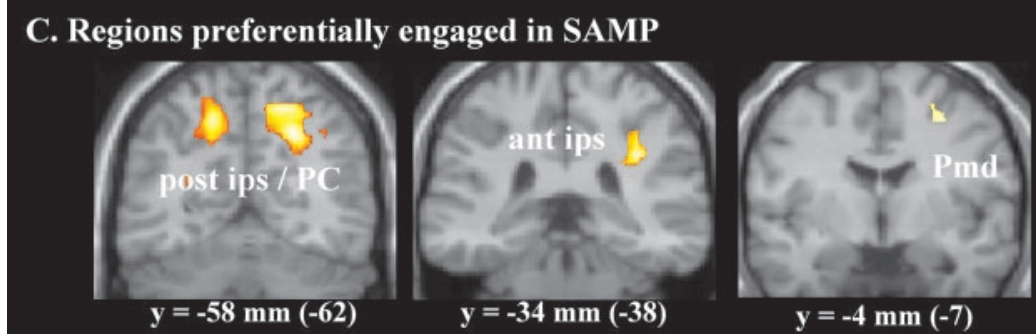

Z-score

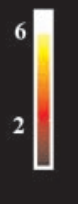

D. Regions preferentially engaged in MPP


FIG. 2. Functional MRI results. A: activation patterns shown on lateral and medial views of the MNI brain template, separately for the spatial attention and memory paradigm (SAMP, red) and for the motor preparation paradigm (MPP, yellow). Overlapping activation is shown in orange. The green line outlines the central sulcus (ces), and the dark vertical line indicates the VAC plane in the MNI system of coordinates. $B$ : conjunction analysis. Brain regions similarly engaged in both SAMP and MPP. $C$ : interaction analysis. SAMP-selective activation pattern. $D$ : interaction analysis. MPP-selective activation pattern. E: lateral premotor activation in the 3 subjects (of the 10 included in $A-D$ ) submitted to an oculomotor control paradigm identical to SAMP except for the presence of ocular saccades during the task condition. Areas involved in the control of saccades, as identified by comparison of the 2 paradigms (interaction analysis, blue), are superimposed on SAMP activation pattern for the same 3 subjects (main effect, red). The overlap (purple) thus corresponds to SAMP-activated areas showing a significantly greater activation with the addition of saccades. Conventions as in $A . B, C$, and $D$ : activation is projected on coronal slices from the MNI template, taken at the $y$ coordinate shown below each slice. Number in parentheses indicates the rostrocaudal Talairach coordinate of the pixel showing the local maximum intensity (see Table 4). The color code (right) represents the $Z$-score associated with activated voxels in the shown slices. $\mathrm{CN}$, caudate nucleus; SMA, supplementary motor area; cis, cingulate sulcus; post and ant ips, posterior and anterior intraparietal sulcus; PC, precuneus; PMd, dorsal lateral premotor cortex. Note that the most ventral cluster of MPP-“activated" voxels ( $A$, left medial view) and associated MPP-selective amygdala "activation" (A? in $D$ ) are illustrated for thoroughness, but should be considered with caution given the proneness of the amygdala region to false-positive artifacts (see RESULTS)
INTERACTION ANALYSIS: BRAIN REGIONS PREFERENTIALLY ENGAGED IN SPATIAL ATTENTION AND MEMORY. This analysis revealed a SAMP-selective activation in the dorsal premotor cortex situated within the right superior precentral sulcus. The only other SAMP-selective activation was a widespread bilateral focus centered on the posterior intraparietal sulcus and precuneus, extending into the anterior intraparietal sulcus in the right hemisphere and into the parieto-occipital sulcus in the left hemisphere.

INTERACTION ANALYSIS: BRAIN REGIONS PREFERENTIALLY ENGAGED IN MOTOR PREPARATION. This analysis revealed a MPP-selective activation in the left dorsal premotor cortex situated in the caudalmost portion of the precentral gyrus, possibly extending into M1. The maximum $Z$-score of this MPP-selective left dorsal premotor activation was located 8 $\mathrm{mm}$ posterior to that of the SAMP-selective right dorsal premotor cortex activation (see Table 4). Except for a dubious amygdala activation, the only other MPP-selective activation focus was located in the medial premotor cortex, involving the medial frontal gyrus caudal to the VAC plane (i.e., the SMA according to Picard and Strick 1996) and the cingulate sulcus.

OCULOMOTOR REGIONS AND SAMP ACTIVATION IN LATERAL PREMOTOR CORTEX. The interaction analysis for three subjects identified a lateral premotor site, located in and near the precentral sulcus (Table 5), that was significantly more active when the spatial attention and memory task was performed with saccades (oculomotor control paradigm) than without saccades (covert shifts of attention, SAMP). The left activation peak was more posterior and ventral than the right one (Talairach $y, z:-11,42$ vs. $-3,52$ ), but both peaks remained within the range of published coordinates for the human FEF, which includes $y, z$ : $-19,47$ (Petit et al. 1997), -8, 54 (Beauchamp et al. 2001), and -6-1, 44-51 (Paus 1996). When superimposed to the SAMP activation map for the same three subjects (Fig. 2E), saccade-related activation on the right hemisphere overlapped with the caudal portion of SAMP premotor activation, whereas the left hemisphere activation was found largely caudal and ventral to it. 
TABLE 2. Activation pattern obtained with the spatial attention/memory paradigm



For each cluster, the region showing the maximum $Z$-score is listed first, followed by the other regions belonging to the cluster [in brackets]. The statistical significance threshold for individual voxels was set at $Z=3.09(P<0.001) . x, y$, and $z$ indicate respectively the left-right, rostro-caudal, and ventro-dorsal Talairach coordinates. $P$ is the statistical significance of the activation, $k$ the number of voxels in the cluster, and $n$ the number of subjects for whom the region was found activated in individual analyses. L, left hemisphere; R, right hemisphere; BA, Brodmann area; PMd, dorsal premotor cortex; SMA, supplementary motor area.

\section{I S C U S S I O N}

The present experiment was designed to compare, in the same subjects, the involvement of the dorsal premotor cortex in two functional domains: spatial attention and memory and motor preparation. The key finding was that each of these domains recruited a different region within the dorsal premotor cortex, as predicted from monkey physiology. The motor preparation paradigm preferentially engaged a caudal portion of PMd, located in the precentral gyrus, whereas the spatial attention/memory paradigm preferentially activated a more rostral region, in and anterior to the precentral sulcus. Outside the lateral premotor cortex, the pre-SMA and caudate nucleus were found to be activated during both paradigms, whereas the parietal cortex was selectively activated by spatial attention/memory, and the SMA by motor preparation. In this section, we will first evaluate insights pro-

TABLE 3. Activation pattern obtained with the motor preparation paradigm

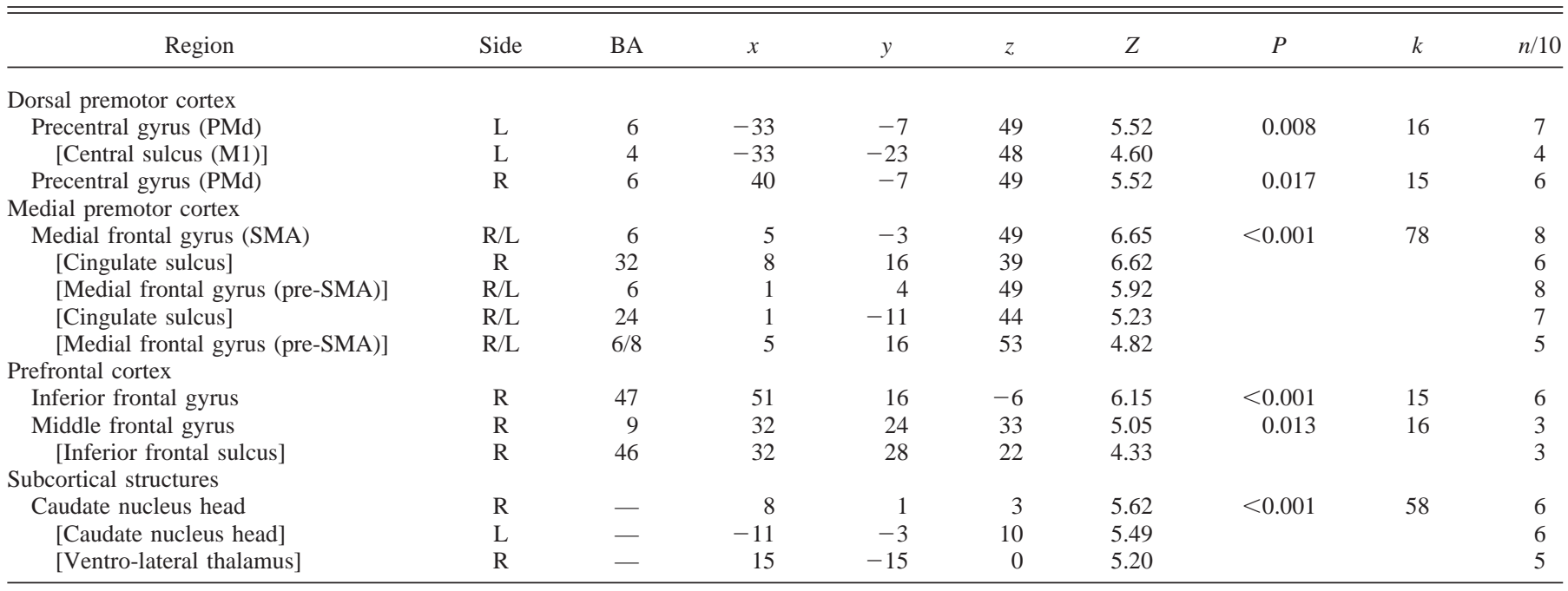

M1, primary motor cortex.

Other conventions as in Table 2. 


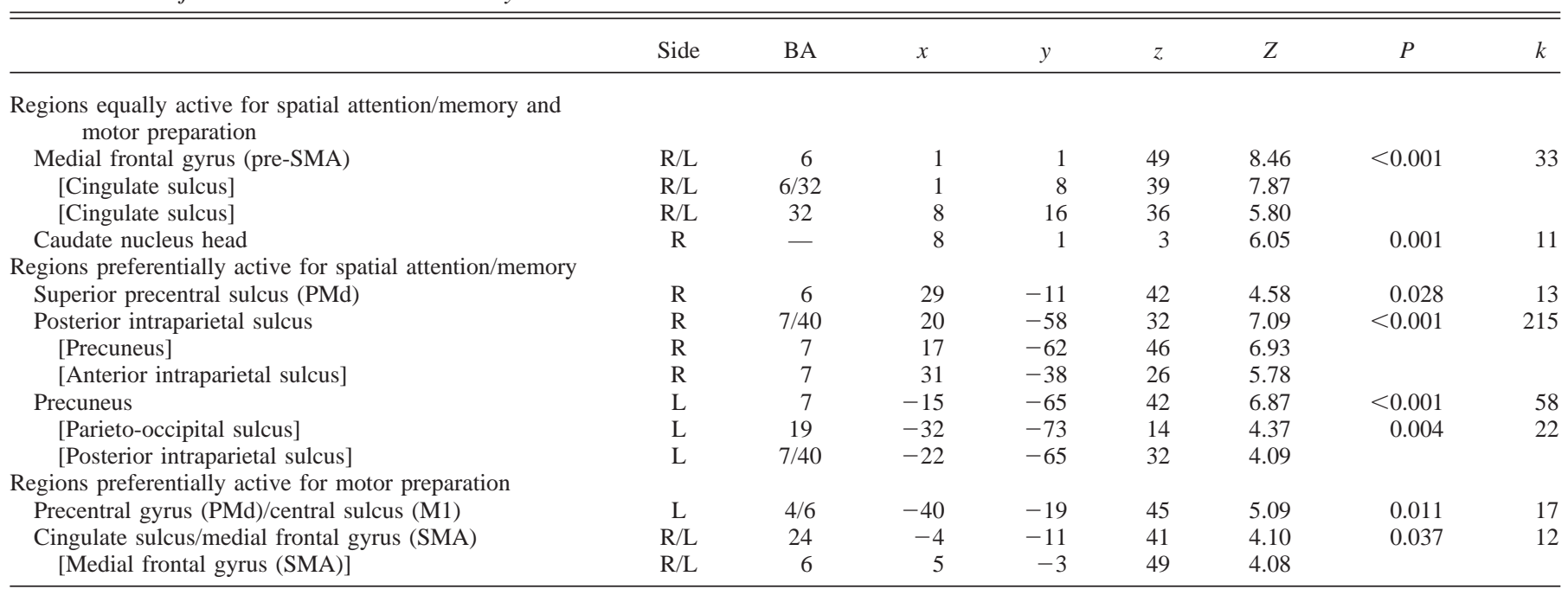

Conventions as in Tables 2 and 3.

vided by each paradigm relative to earlier imaging studies of either spatial attention/memory or motor preparation; then, we will turn to evidence from nonhuman primates to discuss the neuroanatomical overlap and segregation of these two functional domains.

\section{SAMP}

A considerable number of earlier neuroimaging studies have explored the neural bases of short-term maintenance of spatial information in working memory (see e.g., Haxby et al. 2000; Smith and Jonides 1999, for reviews), or those of spatial attentional shifts, whether overt or covert (i.e., with or without ocular saccades; see e.g., Beauchamp et al. 2001; Corbetta et al. 1998; Coull and Nobre 1998; Kawashima et al. 1998; Nobre et al. 2000; Petit et al. 1996). All these studies emphasize the involvement of a distributed parieto-frontal network which resembles that yielded by the (task - control) subtraction in our (covert) spatial attention and memory paradigm. This network includes the dorsal premotor cortex, as well as the intraparietal sulcus and precuneus, the lateral prefrontal cortex, and the pre-SMA and underlying cingulate sulcus (for pre-
SMA/SMA distinction in monkeys and humans, see Tanji 1994 and Picard and Strick 1996, respectively).

In dorsal premotor cortex, SAMP activation occupied a large area involving both the superior precentral and superior frontal sulci. Since our task purposely conflated covert attentional shifts and working memory (which are both stimulus-oriented as opposed to movement-oriented), the respective contribution of each function cannot be determined. However, the results of our oculomotor control paradigm converge with previous imaging data to suggest that SAMP activation encompassed at least two different subregions. Imaging studies of eye movements have localized the human FEF to the superior precentral sulcus (e.g., Lobel et al. 2001; Paus 1996; Rosano et al. 2002) and demonstrated that it is activated not only by actual saccades, but also by covert attentional shifts (Corbetta et al. 1998; Gitelman et al. 1999), albeit to a lesser magnitude (Beauchamp et al. 2001). Accordingly, SAMP activation in lateral premotor cortex was found to overlap with areas whose activity was increased by ocular saccades, particularly in the right hemisphere. Nevertheless, SAMP activation largely extended, in both hemispheres, into the adjacent superior frontal sulcus or gyrus. This region coincides with the focus, rostrally distinct

TABLE 5. PMd activation pattern in the three subjects who performed the spatial attention and memory task both without (SAMP) and with (oculomotor control paradigm) ocular saccades



Conventions as in Tables 2 and 3. 
from FEF, reported by previous imaging studies using tasks that require item maintenance in spatial working memory (e.g., Courtney et al. 1998; LaBar et al. 1999; Merriam et al. 2001; Pochon et al. 2001; Rowe and Passingham 2001; Rowe et al. 2000). Taken together, the present and earlier findings suggest that SAMP activation in lateral premotor cortex involved the FEF, at least partly, but engaged in addition, a rostrally adjacent subregion contributing to memory, attention, or both. This conclusion is consistent with recording data in monkeys demonstrating attentional/mnemonic neural properties in rostral $\mathrm{PMd}$, a region that is clearly separate from the FEF (Boussaoud 2001; Lebedev and Wise 2001).

Notwithstanding the above limitation, our results strengthen other authors' conclusions (Smith and Jonides 1999; Ungerleider et al. 1998) that the dorsal premotor activation that has routinely been described in spatial attention or memory imaging studies cannot be dismissed as reflecting incidental motor processes. SAMP control condition provided a stringent baseline that was equated with the task for motor components. First, movement selection and preparation occurred at the same time and followed the same rule in task and control. Second, the two conditions necessitated the execution of an equal number of index and middle finger responses. Third, motor preparedness (i.e., general readiness to select, prepare, and execute a movement) was similarly present in both conditions. As a result, all motor processes were presumably subtracted in our paradigm, and the resulting dorsal premotor activation can reasonably be considered as reflecting on-line covert attentional shifts and/or short-term maintenance of visuospatial information relevant for action.

\section{$M P P$}

In this study, activation related to motor preparation was assessed by comparing deferred versus immediate execution of a single visually instructed finger movement. The subjects' markedly faster reaction times during MPP task relative to control (average gain of time: $283 \mathrm{~ms}$ ) demonstrated that motor preparation did occur during the extended delays. MPP (task control) contrast outlined a frontal cortical network including the caudal portion of the dorsal premotor cortex, bilaterally, the primary motor cortex contralateral to the moving finger, and the medial premotor cortex (both the pre-SMA and SMA regions and the underlying cingulate sulcus). These three motor regions were also found to be activated in previous studies of protracted motor preparation of a single visually guided movement using positron emission tomography (Deiber et al. 1996; Krams et al. 1998; Rushworth et al. 2001), or eventrelated fMRI (Lee et al. 1999; Toni et al. 1999). However, unlike earlier studies, we found a circumscribed dorsal premotor activation, centered on the caudal portion of this cortical region, and we did not observe any significant activation in the parietal cortex. These differences may be due to particularities of the present behavioral paradigm.

Since our objective was to distinguish premotor involvement in attention/memory versus movement preparation, the MPP subtraction was specifically designed to exclude all the stimulus-related components inherent in delayed visually guided responses. First, visuospatial input was strictly identical in MPP task and control. Second, the subjects' gaze and attention were similarly focused on the central cross during the task's long preparatory periods and corresponding control intervals. Third, to avoid taxing working memory (i.e., retrospective stimulus representation, as opposed to the targeted prospective movement coding), the instructing stimulus (MIC) remained present during the entire preparatory period, and subjects were explicitly asked to refrain from verbal rehearsing (a pilot investigation having shown that some subjects spontaneously favored this strategy despite MIC continuous presence). The present protocol therefore contrasts with the studies listed above, which generally used brief stimulus presentation followed by "empty" delays during which the subjects' activity was unclear. For example, in their event fMRI study of delayed finger movements, Toni et al. (1999) used briefly presented (300 ms) cues followed by 1.28- to 12.8-s delays. This protocol yielded two distinct dorsal premotor activation foci. The posterior one was related to the movement, consistent with the present results, but the anterior one showed complex signal-, delay-, and movement-related responses, which may reflect unintended recruitment of attentional and/or mnemonic processes. Such recruitment could also explain the supra-threshold involvement of parietal cortex reported in earlier studies, a hypothesis supported by the result of the present interaction analysis which identified the parietal activation as SAMPselective.

\section{Overlap of spatial attention/memory and motor preparation outside the dorsal premotor cortex}

Superimposition of SAMP and MPP activation maps yielded a minimal overlap in the right lateral prefrontal cortex (in the inferior frontal gyrus), and a more extensive one in the caudate nucleus and pre-SMA. The equal involvement of the two latter regions in both tasks was further confirmed by a conjunction analysis. These regions therefore seem to contribute to both spatial attention/memory and motor preparation, without any obvious regional specialization detectable at the voxel level of resolution, at least for the simple expression of these functions measured here, that is, on-line maintenance of a single spatial location and preparation of a single finger movement. The overlap of functions observed in the lateral prefrontal cortex and striatum is in agreement with neurophysiological recordings in monkeys showing that neurons in these two structures possess both attentional/mnemonic and motor preparatory properties (Boussaoud and Kermadi 1997; Boussaoud and Wise 1993a,b; di Pellegrino and Wise 1993; Lebedev and Wise 2001; Quintana and Fuster 1999). Regarding prefrontal cortex, the present results also confirm those of the only previous imaging study that, to our knowledge, has compared sensory memory for a single item versus motor preparation of a single movement in the same set of subjects (D'Esposito et al. 2000). Indeed, as in the present study, no regional selectivity for one or the other function was found in lateral prefrontal cortex. Finally, the difference between the pre-SMA, which was active in both paradigms, and the SMA, which was preferentially involved in MPP, corroborates earlier imaging data indicating that both areas contribute to motor control (e.g., Lee et al. 1999), whereas only the pre-SMA participates in working memory (e.g., Petit et al. 1998). Our results also fit well with current models positing a functional caudo-rostral continuum from motor to cogni- 
tive functions in medial premotor regions (Picard and Strick 1996; Tanji 1996), akin to that proposed below for the lateral aspect of premotor cortex.

\section{Segregation of spatial attention/memory and motor preparation in PMd}

In monkeys, a large body of data indicates that PMd is not a homogeneous area. Anatomically, Barbas and Pandya (1987) have divided PMd into a rostral region (PMdr, approximately corresponding to F7 in Matelli et al. 1985) and a caudal one (PMdc or F2). These two subregions are in receipt of different signals from the posterior parietal cortex. In particular, only PMdr receives strong inputs from visual areas of the parietooccipital region, which seems important for detection of peripheral targets (Marconi et al. 2001; Matelli et al. 1998; Shipp et al. 1998; Tanné et al. 1995). In addition, PMdr is the primary target of prefrontal and FEF inputs, whereas PMdc is the main source of projections to the primary motor cortex and spinal cord (Barbas and Pandya 1987; Dum and Strick 1991; He et al. 1993; Johnson et al. 1996). Accordingly, intracortical microstimulation was found to evoke saccades in PMdr, and arm or body movements in PMdc (Fujii et al. 2000). Thus PMdr is linked to regions known to contribute to memory and spatial attention (which is tightly linked to eye movements, see e.g., Beauchamp et al. 2001), and can in turn affect motor control via its connections with PMdc (Barbas and Pandya 1987; J. Tanné-Gariépy, E. M. Rouiller, and D. Boussaoud, unpublished data).

Anatomical differences between the two subregions are paralleled by differences in neuronal properties. Most PMdc cells are active during arm movements toward a visual target but not during saccades to the same target; by contrast, PMdr contains equal proportions of cells coding saccades to the target, arm movements, or both (Fujii et al. 2000). It is also in PMdr that Lebedev and Wise (2001) found neurons coding the orientation of spatial attention regardless of eye position, saccade direction, or hand movement target. Finally, as mentioned in the INTRODUCTION, recordings during a "SAM/MIC" task, akin to the present MPP task, provided a direct comparison, in the same population of neurons, of attentional/mnemonic (i.e., SAM-related) versus motor preparatory (i.e., MIC-related) properties (see Boussaoud 2001 for review). The results demonstrated that both types of properties were present in $\mathrm{PMd}$, with a higher proportion of attention/memory-related neurons in PMdr relative to PMdc.

In humans, it is not known which anatomical zones correspond to the monkey PMdc and PMdr (Baleydier et al. 1997; Jackson and Husain 1996; Roland and Zilles 1996). Preuss et al. (1996) proposed that PMdr corresponds to area 6 in and rostral to the superior portion of the precentral sulcus, while PMdc occupies much of the precentral gyrus behind it. Our results fit particularly well with this proposal. Indeed, spatial attention and memory activated a region encompassing the superior precentral sulcus and the rostrally adjacent portion of the superior frontal sulcus, whereas motor preparation recruited a more posterior region located within the precentral gyrus. This relative rostro-caudal specialization was confirmed by interaction analyses, which identified a SAMP-selective focus in the right precentral sulcus located $8 \mathrm{~mm}$ anterior to the MPP-selective focus identified in the left precentral gyrus.
These findings provide strong evidence in favor of a parcellation of the human PMd parallel to that observed in monkeys. They suggest, in addition, that in both species, this parcellation underlies a similar functional segregation, with PMdc tightly associated with the control of specific arm or body movements, and PMdr playing a key role in orienting attention to and maintaining visuo-spatial information relevant for goal-directed action, regardless of the motor effector.

One difference, however, is that in the monkey, the PMdr region that presents attentional/mnemonic neural properties is clearly distinct from and posterior to both the FEF and the prefrontal cortex (Boussaoud and Wise 1993a,b; Lebedev and Wise 2001). By contrast, in humans, the region in the superior frontal sulcus recruited by spatial attention and memory is anterior to the functionally defined FEF, a location that makes its designation ambiguous. Namely, some authors attribute it, as we did, to rostral premotor area 6 (e.g., Pochon et al. 2001 for a right focus at Talairach coordinates 27, 3, 51), and others to prefrontal area 8 (e.g., Rowe and Passignham 2001 for a bilateral focus at $28,8,60$ and $-18,0,56)$. The inaccuracy of current methods for cytoarchitectonic labeling of activation foci (see Brett et al. 2002 for an in-depth review) precludes settlement of this issue, which is further complicated by the absence of a sharp border between human areas 6 and 8 (Baleydier et al. 1997). Our conclusion that the activation peaks observed for SAMP $(-20,1,49$, and $29,1,56)$ lie in the human counterpart of the monkey PMdr is thus proposed in a tentative way, pending the development of more accurate localization methods (using probabilistic atlases or in vivo architectonic mapping of the human brain; see Brett et al. 2002). This conclusion is nevertheless congruent with monkey data showing that PMdr, but not area 8, lesions disrupt performance on visuo-motor conditional tasks such as those used here (Petrides 1985).

We thank F. Hadj-Bouziane for thoughtful comments on an earlier version of the manuscript.

This study was supported by the Program Thématiques de la Région RhôneAlpes. S. Simon was supported by the French Ministry of Research.

Present address of A. M. Berardi: Département de Psychologie, Université de Metz, 57045 Metz, France.

\section{REFERENCES}

Baleydier C, Achache P, And Froment JC. Neurofilament architecture of superior and mesial premotor cortex in the human brain. Neuroreport 8 : 1691-1696, 1997.

BARBAS H AND PANDYA DN. Architecture and frontal cortical connections of the premotor cortex (area 6) in the rhesus monkey. J Comp Neurol 256: 211-228, 1987

Beauchamp MS, Petit L, Emmore TM, Ingeholm J, and Haxby JV. A parametric study of overt and covert shifts of visuospatial attention. NeuroImage 14: 310-321, 2001.

BousSAOUd D. Attention versus intention in the primate premotor cortex. NeuroImage 14: S40-S45, 2001.

BoussaOUD D AND KERMADI I. The primate striatum: neuronal activity in relation to spatial attention versus motor preparation. Eur J Neurosci 9: 2152-2168, 1997.

BoussaOUd D AND WiSE SP. Primate frontal cortex: neuronal activity following attentional versus intentional cues. Exp Brain Res 95: 15-27, 1993a.

BoussaOUd D AND WISE SP. Primate frontal cortex: effects of stimulus and movement. Exp Brain Res 95: 28-40, 1993 b.

BretT M, JohnsRude IS, AND OwEn AM. The problem of functional localization in the human brain. Nature Rev Neurosci 3: 243-249, 2002. 
CAMINITI R, FERRAINA S, AND MAYER AB. Visuomotor transformations: early cortical mechanisms of reaching. Curr Opin Neurobiol 8: 753-761, 1998.

Caminiti R, Johnson PB, Galli C, Ferraina S, and Burnod Y. Making arm movements within different parts of space: the premotor and motor cortical representation of a coordinate system for reaching to visual targets. $\mathrm{J} \mathrm{Neu}$ rosci 11: 1182-1197, 1991.

Cohen JD, Mc Whinney B, Flatt M, and Provost J. Psyscope: a new graphic interactive environment for designing psychology experiments. $B e$ hav Res Meth Instr Comp 25: 257-271, 1993.

Corbetta M, Akbudak E, Conturo Te, Snyder AZ, Ollinger JM, Druhy HA, Linenweber MR, Petersen SE, Raichle ME, Van Essen DC, AND SHULMAN GL. A common network of functional areas for attention and eye movements. Neuron 21: 761-773, 1998.

COUll JT AND NoBre AC. Where and when to pay attention: the neural systems for directing attention to spatial locations and to time intervals as revealed by both PET and fMRI. J Neurosci 18: 7426-7435, 1998.

Courtney SM, Petit L, Maisog JM, Ungerleider LG, and Haxby JV. An area specialized for spatial working memory in human frontal cortex. Science 279: 1347-1351, 1998.

CRAmmond DJ AND Kalaska JF. Prior information in motor and premotor cortex: activity during the delay period and effect on pre-movement activity. J Neurophysiol 84: 986-1005, 2000.

Deiber M-P, Ibanez V, Sadato N, and Hallett M. Cerebral structures participating in motor preparation in humans: a positron emission tomography study. J Neurophysiol 75: 233-247, 1996.

Deiber M-P, Wise SP, Honda M, Catalan MJ, Grafman J, and Hallett M. Frontal and parietal networks for conditional motor learning: a positron emission tomography study. J Neurophysiol 78: 977-991, 1997.

D’Esposito M, Aguirre GK, Zarahn E, Ballard D, Shin RK, and Lease J. Functional MRI studies of spatial and nonspatial working memory. Cog Brain Res 7: 1-13, 1998.

D'Esposito M, Ballard D, Zarahn E, and Aguirre GK. The role of the prefrontal cortex in sensory memory and motor preparation: an event-related fMRI study. NeuroImage 11: 400-408, 2000.

Di Pellegrino G and Wise SP. Visuospatial versus visuomotor activity in the premotor and prefrontal cortex of a primate. J Neurosci 13: 1227-1241, 1993.

Dum RP AND STRICK PL. The origin of corticospinal projections from the premotor areas in the frontal lobe. J Neurosci 11: 667-689, 1991.

Evans AC, Collins DL, Mills SR, Brown ED, Kelly RL, and Peters TM. 3D statistical neuroanatomical models from 305 MRI volumes. Proc IEEE Nucl Sci Symp Med Imaging Conf 1813-1817, 1993.

Friston KJ, Ashburner J, Frith CD, Poline J-B, Heather JD, and FraCKOWIAK RSJ. Spatial registration and normalization of images. Hum Brain Mapp 2: 1-25, 1995a.

Friston KJ, Holmes AP, Worsle KJ, Poline J-B, Frith CD, and FrackoWIAK RSJ. Statistical parametric maps in functional imaging: a general linear approach. Hum Brain Mapp 3: 189-210, 1995 b.

Fu QG, Flament D, Coltz JD, and Ebner TJ. Temporal encoding of movements kinematics in the discharge of primate primary motor and premotor neurons. J Neuropshysiol 73: 836-854, 1995.

FuJII N, Mushiake H, AND TANJI J. Rostrocaudal distinction of the dorsal premotor area based on oculomotor involvement. J Neurophysiol 83: 1764 1769, 2000.

Gitelman DR, Nobre AC, Parrish TB, LaBar KS, Kim YH, Meyer JR, AND Mesulam M-M. A large-scale distributed network for covert spatial attention: further anatomical delineation based on stringent behavioural and cognitive controls. Brain 122: 1093-1106, 1999.

Grafton ST, FAgG AH, AND ARbiB MA. Dorsal premotor cortex and conditional movement selection: a PET functional mapping study. J Neurophysiol 79: 1092-1097, 1998.

Halsband U AND FREUnd HJ. Premotor cortex and conditional motor learning in man. Brain 113: 207-222, 1990.

Haxby JV, Petit L, Ungerleider L, And Courtney SM. Distinguishing the functional roles of multiple regions in distributed neural systems for visual working memory. NeuroImage 11: 380-391, 2000.

He SQ, Dum RP, AND STRICK PL. Topographic organization of corticospinal projections from the frontal lobe: motor areas on the lateral surface of the hemisphere. J Neurosci 13: 952-980, 1993.

JACKSON SR AND HuSAIN M. Visuomotor functions of the lateral pre-motor cortex. Curr Opin Neurobiol 6: 788-795, 1996.

Johnson PB, Ferraina S, Bianchi L, And Caminiti R. Cortical networks for visual reaching: physiological and anatomical organization of frontal and parietal lobe arm regions. Cereb Cortex 6: 102-119, 1996.
KaWASHIMA R, Roland PE, AND O'Sullivan RT. Fields in human motor areas involved in preparation for reaching, actual reaching, and visuo-motor learning: a positron emission tomography study. J Neurosci 14: 3462-3474, 1994.

Kawashima R, Satoh K, Goto R, Inoue K, Itoh M, and Fukuda H. The role of the left inferior temporal cortex for visual pattern discrimination-a PET study. Neuroreport 9: 1581-1586, 1998.

Krams M, Rushworth M, Deiber M-P, Frackowiak RSJ, and Passignham RE. The preparation, execution and suppression of copied movements in the human brain. Exp Brain Res 120: 386-398, 1998.

Kurata K AND HofFman DS. Differential effects of muscimol microinjection into dorsal and ventral aspects of the premotor cortex. J Neurophysiol 71: 1151-1164, 1994.

LaBar KS, Gitelman DR, Parrish TB, and Mesulam M-M. Neuroanatomic overlap of working memory and spatial attention networks: a functional MRI comparison within subjects. NeuroImage 10: 695-704, 1999.

LEBEDEV MA AND WISE SP. Tuning for the orientation of spatial attention in dorsal premotor cortex. Eur J Neurosci 13: 1002-1008, 2001.

LeE K-M, Chang K-H, AND RoH J-K. Subregions within the supplementary motor area activated at different stages of movement preparation and execution. NeuroImage 9: 117-123, 1999.

Lobel E, Kahane P, Leonards U, Grosbras M, Lehericy S, Le Bihan D, AND BERTHOZ A. Localization of human frontal eye fields: anatomical and functional findings of functional magnetic resonance imaging and intracerebral electrical stimulation. J Neurosurg 95: 804-815, 2001.

Marconi B, Genovesio A, Battaglia-Mayer A, Ferraina S, Squatrito S, MolinARI M, LACQUANITI F, AND CAMINITI R. Eye-hand coordination during reaching. I. Anatomical relationships between parietal and frontal cortex. Cereb Cortex 11: 513-527, 2001.

Matelli M, Govoni P, Galletti C, Kutz DF, and Luppino G. Superior area 6 afferents from the superior parietal lobule in the macaque monkey. J Comp Neurol 402: 327-352, 1998.

Matelli M, Luppino G, and Rizzolatti G. Patterns of cytochrome oxidase activity in the frontal agranular cortex of the macaque monkey. Behav Brain Res 18: 128-136, 1985

Merboldt K-D, Fransson P, Bruhn H, And Frahm J. Functional MRI of the human amygdala. NeuroImage 14: 253-257, 2001.

Merriam EP, Colby CL, Thulborn KR, Luna B, Olson CR, and Sweeney JA. Stimulus-response incompatibility activates cortex proximate to three eye fields. NeuroImage 13: 794-800, 2001.

Nobre AC, Gitelman DR, Dias EC, And Mesulam M-M. Covert visual spatial orienting and saccades: overlapping neural systems. NeuroImage 11: 210-216, 2000.

OLDFIELD RC. The assessment and analysis of handedness: the Edinburgh inventory. Neuropsychologia 9: 97-113, 1971.

Passingham RE. The Frontal Lobes and Voluntary Action. Oxford, UK: Oxford University Press, 1993, vol. 21.

PAUS T. Location and function of the human frontal eye-field: a selective review. Neuropsychologia 34: 475-483, 1996.

Petit L, Clark VP, Ingeholm J, and Haxby JV. Dissociation of saccaderelated and pursuit-related activation in human frontal eye fields as revealed by fMRI. J Neurophysiol 77: 3386-3390, 1997.

Petit L, Courtney SM, Ungerleider L, And Haxby JV. Sustained activity in the medial wall during working memory delays. J Neurosci 18: 9429-9437, 1998.

Petit L, Orssaud C, Tzourio N, Crivello F, Berthoz A, and Mazoyer B. Functional anatomy of a prelearned sequence of horizontal saccades in humans. J Neurosci 16: 3714-3726, 1996.

Petrides M. Deficits in non-spatial conditional associative learning after periarcuate lesions in the monkey. Behav Brain Res 16: 95-101, 1985.

PETRIDEs M. The effect of periarcuate lesion in the monkey on the performance of symmetrically and asymmetrically reinforced visual and auditory go, no-go tasks. J Neurosci 6: 2054-2063, 1986.

PICARD N AND STRICK PL. Motor areas of the medial wall: a review of their location and functional activation. Cereb Cortex 6: 342-353, 1996.

Pochon JB, Levy R, Poline JB, Crozier S, Lehéricy S, Pillon B, Deewer B, LE BiHAN D, AND Dubois B. The role of dorsolateral prefrontal cortex in the preparation of forthcoming actions: an fMRI study. Cereb Cortex 11: 260-266, 2001.

Preuss TM, StePniewska I, AND KaAs JH. Movement representation in the dorsal and ventral premotor areas of owl monkeys: a microstimulation study. J Comp Neurol 371: 649-676, 1996.

PRICE CJ AND FRISTON KJ. Cognitive conjunction: a new approach to brain activation experiments. NeuroImage 5: 261-270, 1997. 
QuintANA J AND Fuster J. From perception to action: temporal integrative functions of prefrontal and parietal neurons. Cereb Cortex 9: 213-221, 1999.

Richter W, ANDERSen PM, Georgopoulos AP, ANd Kim S-G. Sequential activity in human motor areas during a delayed cued finger movement task studied by time-resolved fMRI. Neuroreport 8: 1257-1261, 1997.

RiEHLE A AND REQUiN J. Monkey primary motor and premotor cortex singlecell activity related to prior information about direction and extend of an intended movement. J Neurophysiol 61: 534-549, 1989.

Roland PE AND ZILLES K. Functions and structures of the motor cortices in humans. Curr Opin Neurobiol 6: 773-781, 1996.

Rosano C, Krisky CM, Welling JS, Eddy WF, Luna B, Thulborn KR, and SweEneY JA. Pursuit and saccadic eye movement subregions in human frontal eye field: a high-resolution fMRI investigation. Cereb Cortex 12: 107-115, 2002.

Rowe JB AND PAssingham RE. Working memory for location and time: activity in prefrontal area 46 relates to selection rather than maintenance in memory. Neurolmage 14: 77-86, 2001.

Rowe JB, Toni I, Josephs O, Frackowiak RS, and Passingham RE. The prefrontal cortex: response selection or maintenance within working memory? Science 288: 1556-1560, 2000.

Rushworth MF, Krams M, and Passignham RE. The attentional role of the left parietal cortex: the distinct lateralization and localization of motor attention in the human brain. $J$ Cogn Neurosci 13: 698-710, 2001.

Shipp S, Blanton M, AND ZeKi S. A visuo-somatomotor pathway through superior parietal cortex in the macaque monkey: cortical connections of areas V6 and V6a. Eur J Neurosci 10: 3171-3193, 1998.

Simon S, Meunier M, Berardi A, Piettre L, Segebarth C, and Boussaoud D. Spatial attention vs. motor preparation. The involvement of premotor cortices as assessed by fMRI. NeuroImage 11: S375, 2000.

Simon S, Meunier M, Berardi A, Piettre L, Segebarth C, Gervais R, and Boussaoud D. Spatial attention and memory versus motor preparation: premotor cortex involvement as revealed by fMRI. Soc Neurosci Abstr (729.5), 2001.

Smith EE AND Jonides J. Storage and executive processes in the frontal lobes. Science 283: 1657-1661, 1999.
Stern Ce, Owen AM, Tracey I, Look RB, Rosen BR, and Petrides M. Activity in ventrolateral and mid-dorsolateral prefrontal cortex during nonspatial visual working memory processing: evidence from functional magnetic resonance imaging. NeuroImage 11: 392-399, 2000.

Talairach J And Tournoux P. Co-planar Stereotaxic Atlas of the Human Brain. New York, NY: Thieme, 1988.

TANII J. New concepts of the supplementary motor area. Curr Opin Neurobiol 6: 782-787, 1996

TANJI J. The supplementary motor area in the cerebral cortex. Neurosci Res 19: 251-268, 1994.

TANnÉ J, Boussaoud D, Zeller N, AND Rouiller EM. Direct visual pathways for reaching movements in the macaque monkey. Neuroreport 7: 267-272, 1995.

Tehovnik EJ, Sommer MA, Chou I-H, Slocum WM, and Shiller PH. Eye fields in the frontal lobes of primates. Brain Res Rev 32: 413-448, 2000.

Toni I, Schluter ND, Josephs O, Friston KJ, and Passingham RE. Signal-, set- and movement-related activity in the human brain: an event-related fMRI study. Cereb Cortex 9: 35-49, 1999.

Toni I, Thoenissen D, AND Zilles K. Movement preparation and motor intention. NeuroImage 14: 110-117, 2001.

Ungerleider LG, Courtney SM, AND HaXby JV. A neural system for human visual working memory. Proc Natl Acad Sci USA 95: 883-890, 1998.

van Oostende S, Hecke PV, Sunaert S, Nuttin B, and Marchal G. fMRI studies of the supplementary motor area and the premotor cortex. NeuroImage 6: 181-190, 1997.

WEINRICH M AND WISE SP. The premotor cortex of the monkey. J Neurosci 2: 1339-1345, 1982.

Wise SP, Boussaoud D, Johnson PB, AND CAMINITI R. Premotor and parietal cortex: corticocortical connectivity and combinatorial computations. Annu Rev Neurosci 20: 25-42, 1997.

Worsley KJ, Evans AC, Marrett S, and Neelin P. A three-dimensional statistical analysis for rCBF activation studies in human brain. $J$ Cereb Blood Flow Metab 12: 900-918, 1992.

WORSLEY KJ AND FRISTON KJ. Analysis of fMRI time-series revisited-again. Neurolmage 2: 173-181, 1995. 